\title{
Hepatocarcinoma fibrolamelar un tumor de adultos jóvenes poco frecuente. Reporte de un caso
}

\section{Case Report of Fibrolamellar Hepatocarcinoma, a Rare Tumor of Young Adults}

\author{
Luis Guillermo Toro Rendón, MD, ${ }^{1}$ Vanessa García, MD, ${ }^{2}$ Juan Camilo Peréz Cadavid, MD, ${ }^{3}$ Sergio Iván Hoyos Duque, MD, ${ }^{4}$ \\ Jaime Fernando Chávez Trujillo, MD, ${ }^{4}$ Juan Ignacio Marín Zuluaga, MD, ${ }^{5}$ Óscar Mauricio Santos, MD, ${ }^{5}$ Octavio German Muñoz, MD, ${ }^{5}$ \\ Juan Carlos Restrepo, MD. ${ }^{6}$
}

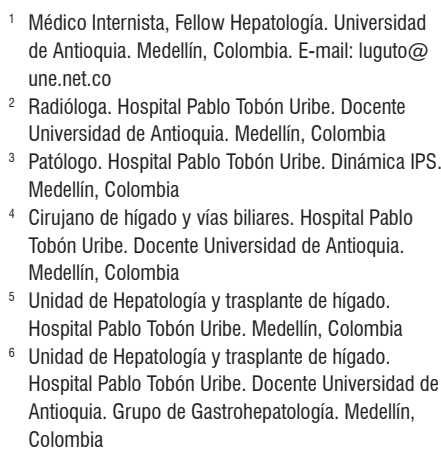

Médico Internista, Fellow Hepatología. Universidad de Antioquia. Medellín, Colombia. E-mail: luguto@ une.net.co

2 Radióloga. Hospital Pablo Tobón Uribe. Docente Universidad de Antioquia. Medellín, Colombia

3 Patólogo. Hospital Pablo Tobón Uribe. Dinámica IPS Medellín, Colombia

${ }^{4}$ Cirujano de hígado y vías biliares. Hospital Pablo Tobón Uribe. Docente Universidad de Antioquia. Medellín, Colombia

5 Unidad de Hepatología y trasplante de hígado. Hospital Pablo Tobón Uribe. Medellín, Colombia

${ }^{6}$ Unidad de Hepatología y trasplante de hígado. Hospital Pablo Tobón Uribe. Docente Universidad de Antioquia. Grupo de Gastrohepatología. Medellín, Colombia

Fecha recibido: $09-01-14$ Fecha aceptado: 05-11-14

\begin{abstract}
Resumen
El hepatocarcinoma fibrolamelar (CHC-FL) es una rara variante del carcinoma hepatocelular (CHC), se presenta con mayor frecuencia en adultos jóvenes, sin distinción de sexo, se desarrolla en su manera más característica sobre un hígado previamente sano. La manifestación clásica está dada por la triada de masa palpable en hipocondrio derecho, dolor y pérdida de peso.

Ante la ausencia de historia de enfermedad hepática previa, es usual que se encuentre como una neoplasia avanzada de gran tamaño, la cual tiene hallazgos característicos en la tomografía o en la RM, que permiten en un alto porcentaje de casos hacer el diagnóstico solo con las imágenes.

En este tipo de neoplasias, el mejor tratamiento es la resección quirúrgica, que proporciona una sobrevida a 5 años de 58-82\%, pero con unas tasas de recaídas muy elevadas, que puede variar según las series entre $33-100 \%$ de los casos.

Presentamos el caso de un paciente joven, sin historia de enfermedad hepática, en el que se diagnosticó un CHC-FL mediante el uso de resonancia magnética con medio de contraste órgano-específico y que es tratado con resección quirúrgica radical.
\end{abstract}

\section{Palabras clave}

Hepatocarcinoma fibrolamelar, carcinoma hepatocelular, hepatectomía, neoplasia hepática.

\section{Abstract}

Fibrolamellar hepatocellular carcinoma (FL-HCC) is a rare variant of hepatocellular carcinoma (HCC) which occurs most often in young adults without regard to sex. It develops in its characteristic way on a previously healthy liver. The classical presentation is a palpable mass in the right upper quadrant, pain, and weight loss.

In the absence of a prior history of liver disease, it is usual to find a large advanced neoplasia which has characteristic findings in a CT scan or MRI. A high percentage of cases can be diagnosed solely with images.

The best treatment for this type of tumor is surgical resection which provides a 5 -year survival rate of $58 \%$ to $82 \%$ but with very high rates of relapse. In published studies rates of relapse vary between $33 \%$ and $100 \%$.

We report the case of a young patient with no history of liver disease in which fibrolamellar hepatocellular carcinoma was diagnosed using contrast organ-specific MRI. The tumor was treated with radical surgical resection.

\section{Keywords}

Fibrolamellar hepatocellular carcinoma, hepatocellular carcinoma, hepatectomy, hepatic neoplasia. 


\section{DESCRIPCIÓN DEL CASO}

Paciente sexo masculino, 26 años de edad, quien hace seis meses después de consumo abundante de licor presenta malestar general y fiebre, consulta en segundo nivel de atención, donde realizan paraclínicos, dentro de los cuales llama la atención la presencia de transaminasas elevadas (4 veces el valor normal), se realiza ecografía abdominal que demuestra una lesión hepática de 9,8 9 9,3 x 6,3 cm sugestiva de una hiperplasia nodular focal (HNF); es remitido al servicio de hepatología donde se ordena una resonancia magnética (RM) de abdomen contrastada, la cual informa que si bien la lesión podría corresponder a una HNF, no se descarta la posibilidad de un CHC. Ante la duda diagnóstica y las potenciales consecuencias de una biopsia hepática (diseminación del CHC en el tracto de punción, sangrado y bajo rendimiento en el diagnóstico de la HNF), y ante la disponibilidad de medio de contraste órgano-específico, se realiza una nueva RM con ácido gadoxético que reporta una lesión heterogénea, de contornos lobulados, encapsulada y bien circunscrita, que compromete el segmento lateral del hígado, con una cicatriz central radiada y septos hacia la periferia, hipodensa en todas las secuencias, con restricción en las imágenes de difusión y áreas focales de necrosis. En la fase hepatobiliar hay realce solo de la cápsula, sin captación de la masa tumoral, hallazgos que favorecen el diagnóstico de CHC-FL.

Se realizó estadificación, descartando compromiso metastásico y se programó para hepatectomía izquierda con intención curativa. En cirugía se encuentra un tumor hepático gigante que compromete los segmentos II, III, IVA, IVB y parcialmente el V y el VIII, sin evidencia de metástasis hepáticas o peritoneales, se realiza una hepatectomía extendida con vaciamiento linfático del hilio, sin poder preservar la vena hepática media por el plano de transección.

La histopatología reportó un CHC-FL con un tamaño de $15 \mathrm{~cm}$ en su diámetro mayor, clasificación $3 / 4$ de Edmondson y Steiner, sin compromiso metastásico ganglionar.

\section{INTRODUCCIÓN}

El CHC-FL es una variante rara de el CHC, inicialmente descrito por Edmondson en 1956; (1) ya en 1980 varios reportes lo establecen como una entidad diferente (2). Representa menos de 1\% de todas las neoplasias primarias del hígado. Hashem, et al en un análisis retrospectivo entre 1986-2000 de los registros poblacionales de cáncer del programa de vigilancia, epidemiología y resultados finales (SEER), encontraron que el CHC-FL constituyo 0,85\% de todas las neoplasias primarias hepáticas (3). Da cuenta de $0,6-8,6 \%$ de todos los $\mathrm{CHC}$ según diferentes reportes (4). Afecta a ambos géneros por igual $(3,5)$, predomina en adultos jóvenes ( $<40$ años) sin antecedente de enfermedad hepática, con una edad media de 25 años; estudios poblacionales han informado que solo $6 \%$ de los pacientes con CHC-FL son mayores de 50 años, mientras que más de $85 \%$ son $\leq 35$ años, muy diferente a la epidemiología del $\mathrm{CHC}$ donde la incidencia del tumor aumenta con la edad, tiene su pico a los 70 años, es $4-8$ veces más frecuente en hombres y en $90 \%$ de los casos se asocia a un factor de riesgo conocido, siendo los más importantes la presencia de cirrosis de cualquier etiología y la infección por el virus de la hepatitis $B(6,7)$.

La presentación usual del CHC-FL es la de una masa tumoral única en un hígado no cirrótico, no asociada a hepatitis viral, rodeado de una cápsula fibrosa y que puede presentar diseminación metastásica linfática regional (4).

La etiología del CHC-FL es desconocida, no se ha identificado la presencia de un precursor histológico para su desarrollo (8); Cieply et al reportaron una regulación positiva de la $\beta$-catenina por fosforilación de los residuos de tirosina en este grupo de pacientes. Las vías de señalización de la $\beta$-catenina cumplen una multitud de funciones en la biología hepática, algunas de las cuales son la de regular la proliferación de los hepatoblastos, su adecuada diferenciación y el control de algunos de sus procesos metabólicos. Se ha demostrado que la $\beta$-catenina se puede activar vía fosforilación de la tirosina; el estudio en mención sugiere una activación aberrante de esta vía, la cual podría ocasionar alteración de la regulación de diferentes genes, muchos de los cuales juegan un papel importante en la patogénesis tumoral $(9,10)$.

\section{PRESENTACIÓN CLÍNICA}

La triada clásica de esta entidad es la de masa palpable en hipocondrio derecho, dolor y pérdida de peso; aunque usualmente se presenta con síntomas inespecíficos como sensación de plenitud abdominal, anorexia, náuseas y fiebre. La ictericia se presenta en $40 \%$ de los casos, hay reportes de presentaciones poco frecuentes como síndrome de Budd Chiari, hemoperitoneo, compromiso obstructivo biliar, ginecomastia (debido a la producción de aromatasa por las células neoplásicas llevando a la conversión de andrógenos a estrógenos), metástasis ósea, falla hepática aguda y síndromes paraneoplásicos como por ejemplo hipoglucemia, hipertiroidismo y trombosis venosa profunda $(5,11,12)$.

En un reporte publicado por Stevens en 1995, al momento del diagnóstico $70 \%$ de los pacientes presentaban diseminación ganglionar, (13) siendo el compromiso metastásico a nivel del hilio hepático el más frecuente (14). 


\section{DIAGNÓSTICO}

El diagnóstico de CHC-FL se puede realizar por las características imagenológicas del tumor en la tomografía o en la RM, en los casos en los cuales este no es claro, la biopsia con aguja fina o laparoscópica puede ser útil (15).

Dentro de los diagnósticos diferenciales se debe considerar la HNF, CHC, adenoma hepático, hemangioma, carcinoma adenoescamoso, carcinoma de vesícula con extensión al hígado, tumores metastásicos asociados con fibrosis (mama, carcinoides, colangiocarcinoma y tiroides) entre otros (5).

\section{LABORATORIO}

A diferencia de los que sucede en el $\mathrm{CHC}$ donde la alfafetoproteína usualmente se encuentra elevada, solo en $10-15 \%$ de los pacientes con CHC-FL se encuentran valores anormales de esta, que por lo regular no superan los 200 $\mathrm{ng} / \mathrm{ml}$. Se han reportado elevaciones en la neurotensina, la des-gamma-carboxi protrombina y en la capacidad de unión de la vitamina B12, (15) esta última puede ser utilizada para monitorizar la progresión de los pacientes.

\section{CARACTERÍSTICAS IMAGENOLÓGICAS}

El CHC-FL puede ser detectado en una radiografía de abdomen, en la cual aparece como una masa hepática grande o una calcificación en hipocondrio derecho, nodular o estrellada y pequeña en relación al tamaño del tumor (16).

A la ecografía se presenta como una masa única, bien definida, que puede ser lobulada y presentar una ecogenicidad mixta en $60 \%$ de los casos, con un predominio de componentes iso e hiperecoicos. La cicatriz central se puede ver como un área de hiperecogenicidad, que solo se demuestra en 33-60\% de los casos (17).

En la tomografía no contrastada, muchos CHC-FL son lesiones hipodensas cuando se comparan con el tejido hepático. En este tipo de imágenes es mucho más evidente la calcificación cuando está presente en una cicatriz central.

La RM demuestra una lesión focal grande, bien delimitada, con una baja señal de intensidad en T1 y alta en las imágenes de T2. Presenta captación temprana y heterogénea del contraste, la cual desaparece en imágenes más tardías (18). Cuando hay dudas en el diagnóstico se puede realizar una RM con medio de contraste órgano específico, que produce un intenso realce del parénquima hepático en la fase celular, en la cual las lesiones de naturaleza no hepatocitaria no captan el contraste, proporcionando una mejor caracterización de las lesiones. Una particularidad importante es la presencia de una cicatriz central que está compuesta de tejido fibroso y se encuentra en $70 \%$ de los casos (19), puede ser estrellada o amorfa, hipointensa al tejido tumoral que la rodea en las imágenes no contrastadas y en la fase arterial (figura 1), esto ayuda a diferenciarla de la cicatriz de la HNF. En las imágenes tardías la cicatriz central tiene un realce mínimo en 50\% de los casos (20).

\section{PATOLOGÍA}

Macroscópicamente es una masa única en $56 \%$ de los casos, que en $66 \%$ compromete el lóbulo izquierdo, bien definida, no encapsulada. Al corte es de consistencia sólida, bordes irregulares, color gris y marrón; verde cuando la producción de bilis es prominente. Presenta múltiples septos fibrosos que subdividen la masa en lóbulos y que pueden conectarse con una cicatriz central (figura 2), esta recuerda y puede causar confusión con una HNF, se pueden encon-

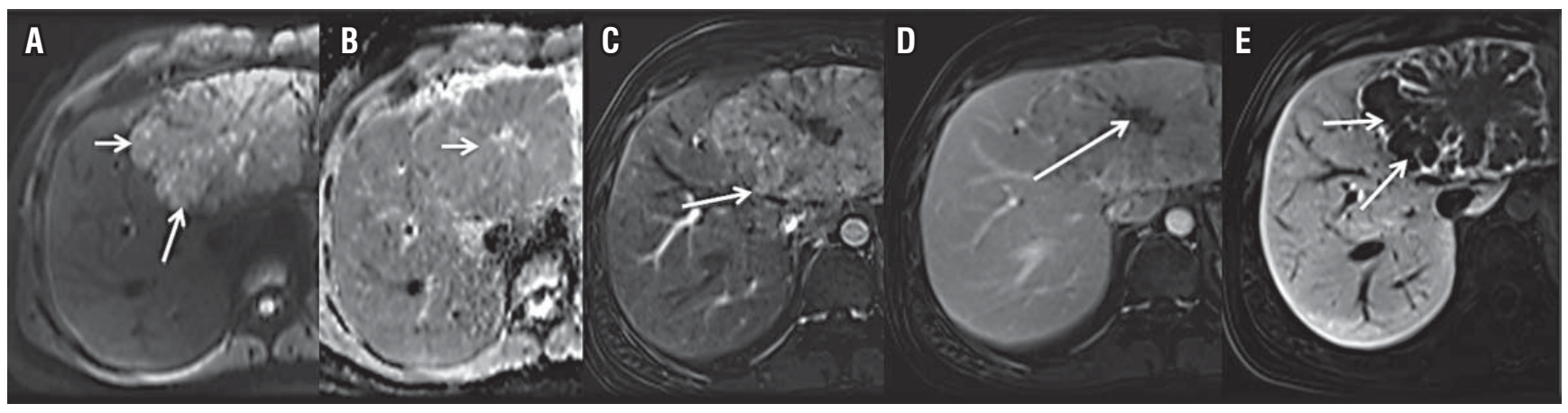

Figura 1. Hallazgos del CHC-FL a la RM. A. Lesión de alta intensidad de señal en las imágenes potenciadas en T2. B. Restricción en la difusión. Una cicatriz que irradia el centro, heterogénea hipo e hiperintensa. C. Imagen dinámica con contraste extracelular, hay captación heterogénea del contraste tempranamente (fase arterial) que se desvanece en las imágenes siguientes. D. Fase portal: la cicatriz tiene mínimo o ningún realce con el contraste, siendo hipointensa con respecto al resto del hígado. E. Con el medio de contraste órgano-específico (ácido gadoxético) en la fase hepatocelular no se demuestra toma del contraste, lesión hipointensa que la diferencia de la HNF atípica. El CHC-FL puede tener realce periférico de la cápsula y de los septos, como en este caso. 
trar calcificaciones en la cicatriz central. El parénquima hepático no neoplásico es de apariencia usual, sin evidencia de fibrosis o cirrosis, aunque la coexistencia de cirrosis se ha reportado en $<4 \%$.

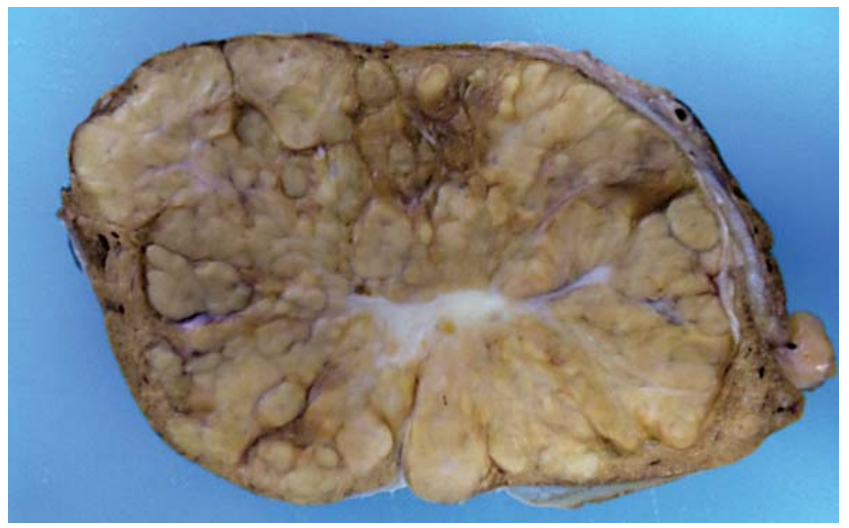

Figura 2. Hallazgos macroscópicos del CHC-FL. Gran masa de apariencia nodular irregular, de $15 \mathrm{~cm}$ de diámetro mayor, compuesta por nódulos de aspecto sólido de color crema, amarillo y verde. Cicatriz central fibrosa característica.

A la histología hay células tumorales grandes, poligonales con citoplasma profundamente eosinófilo, macronucléolos y abundante estroma fibroso dispuesto alrededor de las células tumorales, del que recibe el término fibrolamelar $(4,21)$. En $75 \%$ de los casos, estas bandas fibróticas se unen formando la cicatriz central, asociada a la presencia de bandas radiadas, que se pueden encontrar también en las lesiones metastásicas (6) (figuras 3 y 4 ).

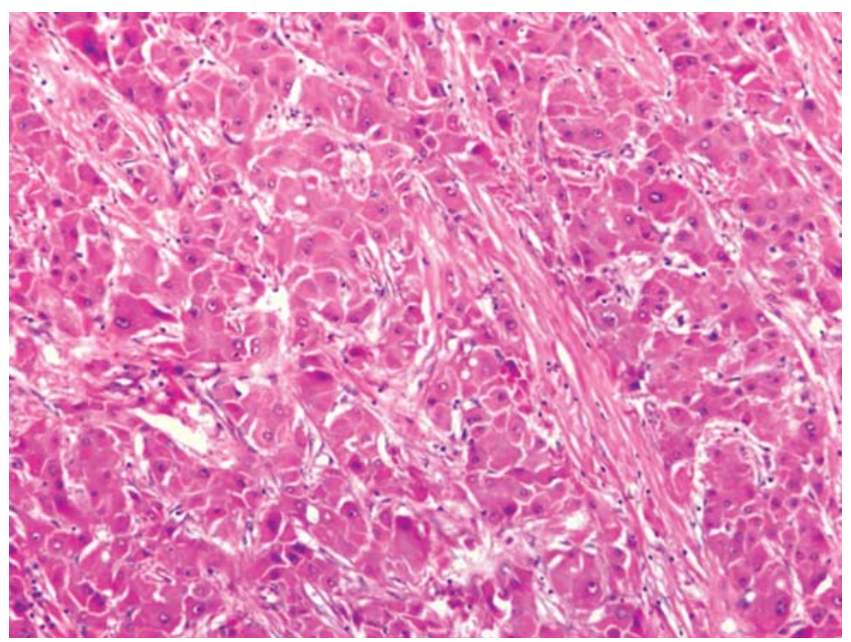

Figura 3. Hallazgos histopatológicos del CHC-FL (hematoxilina y eosina). Se identifican nidos de hepatocitos neoplásicos separados por bandas de tejido fibroconectivo. Los hepatocitos son de gran tamaño, poligonales con citoplasma eosinófilo amplio, ligero pleomorfismo nuclear y nucléolo evidente.

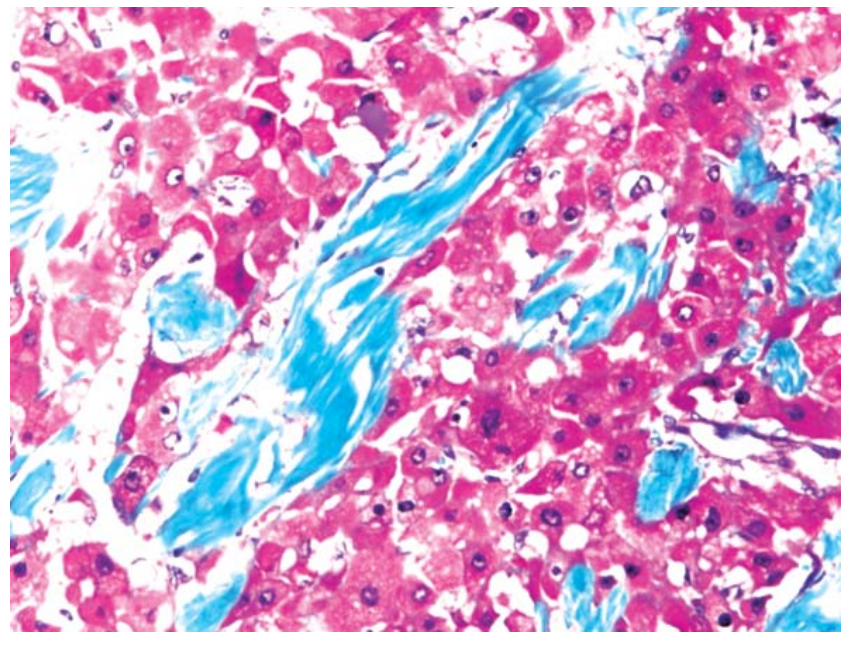

Figura 4. Hallazgos histopatológicos del CHC-FL (tricrómico). Esta tinción resalta las bandas de tejido fibroconectivo que separan los nidos de hepatocitos neoplásicos.

La apariencia eosinofílica de las células tumorales es secundaria a la proliferación mitocondrial (22), 50\% de las veces se pueden encontrar cuerpos hialinos e inclusiones citoplasmáticas llamadas "cuerpos pálidos", estos últimos cuya composición no está bien definida, pero son inmunorreactivos para el fibrinógeno. Es muy raro encontrar esteatosis microvesicular y las figuras mitóticas son menos comunes que en el CHC (16).

Es habitual encontrar en la histología pequeñas calcificaciones, las cuales usualmente se depositan en la cicatriz central o en las bandas fibróticas (16).

La invasión vascular está presente en $40 \%$ de estos tumores (12), 50\% tienen cápsula, la cual es incompleta en la gran mayoría de ellos.

Se pueden encontrar depósito de cobre, que se explica por la presencia de colestasis, común en este tipo de tumores, además se ha reportado una semejanza estructural con las neoplasias neuroendocrinas (4).

Es poco frecuente la coexistencia de CHC y CHC-FL en el mismo paciente y cuando se encuentran, los dos componentes son claramente separados; en el reporte de Stirpa, et al 7\% de los casos de CHC-FL (3/41) presentaban zonas características de $\mathrm{CHC}$ (13).

\section{TRATAMIENTO}

Los CHC-FL son de manera característica neoplasias avanzadas al momento del diagnóstico, los datos sobre el tratamiento y pronóstico son escasos, debido a la baja incidencia de este tumor y a que la información de la que se dispone proviene de series de casos y de cohortes pequeñas.

Las primeras series publicadas en los años 80 describían el CHC-FL como un neoplasia con una tasa de crecimiento 
lento y un pronóstico más favorable que el $\mathrm{CHC}$ (2), estudios recientes demuestran que realmente el CHC-FL no tiene un pronóstico tan favorable como se creía (23).

En una revisión sistemática publicada por Mavros, et al en 2012, se encontró que la sobrevida a 1,3 y 5 años después de hepatectomía era de $93 \%, 80 \%$ y $70 \%$, con una media de 222 meses, en los pacientes a los que se les realizaba trasplante hepático la sobrevida fue de $86 \%, 42 \%$ y $34 \%$, con una media de 32 meses y en aquellos que se consideraron no candidatos a ninguno de los dos de $81 \%, 12 \%$ y $0 \%$ respectivamente, con una media de sobrevida de 20 meses (24).

Con los datos que disponemos hasta ahora el mejor tratamiento es la resección quirúrgica, cuando esta sea posible, la cual provee una sobrevida a 5 años de $58-82 \%$ (25), estas altas tasas podrían estar influenciadas por enfermedad menos avanzada en este grupo de pacientes, cuando son comparados con aquellos que fueron llevados a trasplante y de manera lógica con los que no son tributarios a ningún manejo.

La recurrencia después de realizar una resección con intención curativa es frecuente, en las series las tasas de recaída fluctúan entre 36-100\%, con una media de 10-33 meses $(5,15,25,26)$.

Los factores que se han asociado con pobre sobrevida son el sexo femenino, el estado avanzado de la enfermedad, la presencia de compromiso tumoral ganglionar, la invasión macrovascular y la enfermedad irresecable (4).

En los casos de enfermedad avanzada, no se dispone de esquemas de quimioterapia establecidos, como resultado de la falta de ensayos clínicos específicos. Hasta el momento no se ha demostrado la utilidad de la quimioterapia como tratamiento neoadyuvante, adyuvante o paliativo y su uso no es estándar. Hay reportes del uso de gemcitabina y oxaliplatino (27), cisplatino, vincristina y 5 -fluorouracilo (7), cisplatino y doxorrubicina (7), 5-fluorouracilo e interferón alfa- $2 \mathrm{~b}$ (28), entre otros, con tasas variables de respuesta. Tampoco hay información del uso de sorafenib en este grupo de pacientes, ya que la mayoría de los estudios clínicos en pacientes con CHC, el CHC-FL hace parte de los criterios de exclusión (29).

Como conclusión hay que decir que a diferencia del CHC, más pacientes con CHC-FL son tributarios de resección quirúrgica, esto influenciado por la edad, la ausencia de cirrosis y de hipertensión portal. El factor pronóstico más importante es la posibilidad de realizar una resección quirúrgica, lo cual proporciona una sobrevida promedio a 5 años de $70 \%$, aunque con unas altas tasas de recaída que hacen necesario nuevas estrategias y terapias para prevenir la recidiva de la enfermedad.

\section{REFERENCIAS}

1. Edmondson HA. Differential diagnosis of tumors and tumor-like lesions of liver in infancy and childhood. AMA journal of diseases of children 1956; 91(2): 168-86.

2. Craig JR, Peters RL, Edmondson HA, Omata M. Fibrolamellar carcinoma of the liver: a tumor of adolescents and young adults with distinctive clinico-pathologic features. Cancer 1980; 46(2): 372-9.

3. El-Serag HB, Davila JA. Is fibrolamellar carcinoma different from hepatocellular carcinoma? A US population-based study. Hepatology 2004; 39(3): 798-803.

4. Ang CS, Kelley RK, Choti MA, Cosgrove DP, Chou JF, Klimstra D, et al. Clinicopathologic characteristics and survival outcomes of patients with fibrolamellar carcinoma: data from the fibrolamellar carcinoma consortium. Gastrointestinal cancer research: GCR 2013; 6(1): 3-9.

5. Liu S, Chan KW, Wang B, Qiao L. Fibrolamellar hepatocellular carcinoma. The American journal of gastroenterology 2009; 104(10): 2617-24; quiz 25.

6. EASL-EORTC clinical practice guidelines: management of hepatocellular carcinoma. Journal of hepatology 2012; 56(4): 908-43.

7. Katzenstein $\mathrm{HM}$, Krailo MD, Malogolowkin $\mathrm{MH}$, Ortega JA, Qu W, Douglass EC, et al. Fibrolamellar hepatocellular carcinoma in children and adolescents. Cancer 2003; 97(8): 2006-12.

8. Klein WM, Molmenti EP, Colombani PM, Grover DS, Schwarz KB, Boitnott J, et al. Primary liver carcinoma arising in people younger than 30 years. American journal of clinical pathology 2005; 124(4): 512-8.

9. Cieply B, Zeng G, Proverbs-Singh T, Geller DA, Monga SP. Unique phenotype of hepatocellular cancers with exon-3 mutations in beta-catenin gene. Hepatology 2009; 49(3): 821-31.

10. White BD, Chien AJ, Dawson DW. Dysregulation of Wnt/beta-catenin signaling in gastrointestinal cancers. Gastroenterology 2012; 142(2): 219-32.

11. Rageshree Ramachandran SK. Fibrolamellar hepatocellular carcinoma. Diagnostic Histopathology 2010; 16(8): 38896.

12. Asrani SK, LaRusso NF. Fibrolamellar hepatocellular carcinoma presenting with Budd-Chiari syndrome, right atrial thrombus, and pulmonary emboli. Hepatology 2012; 55(3): 977-8.

13. Stevens WR, Johnson CD, Stephens DH, Nagorney DM. Fibrolamellar hepatocellular carcinoma: stage at presentation and results of aggressive surgical management. AJR American journal of roentgenology 1995; 164(5): 1153-8.

14. Chun YS, Zimmitti G. Fibrolamellar variant of hepatocellular carcinoma. Recent results in cancer research Fortschritte der Krebsforschung. Progres dans les recherches sur le cancer 2013; 190: 101-10. 
15. Stipa F, Yoon SS, Liau KH, Fong Y, Jarnagin WR, D’Angelica $\mathrm{M}$, et al. Outcome of patients with fibrolamellar hepatocellular carcinoma. Cancer 2006; 106(6): 1331-8.

16. Friedman AC, Lichtenstein JE, Goodman Z, Fishman EK, Siegelman SS, Dachman AH. Fibrolamellar hepatocellular carcinoma. Radiology 1985; 157(3): 583-7.

17. McLarney JK, Rucker PT, Bender GN, Goodman ZD, Kashitani N, Ros PR. Fibrolamellar carcinoma of the liver: radiologic-pathologic correlation. Radiographics : a review publication of the Radiological Society of North America, Inc 1999; 19(2): 453-71.

18. Elsayes KM, Narra VR, Yin Y, Mukundan G, Lammle M, Brown JJ. Focal hepatic lesions: diagnostic value of enhancement pattern approach with contrast-enhanced 3D gradient-echo MR imaging. Radiographics: a review publication of the Radiological Society of North America, Inc 2005; 25(5): 1299-320.

19. Ichikawa T, Federle MP, Grazioli L, Madariaga J, Nalesnik M, Marsh W. Fibrolamellar hepatocellular carcinoma: imaging and pathologic findings in 31 recent cases. Radiology 1999; 213(2): 352-61.

20. Elsayes KM, Peterson CM, Menias CO. The central scar: pathophysiology and imaging features. Current problems in diagnostic radiology 2007; 36(6): 247-57.

21. Kakar S, Burgart LJ, Batts KP, Garcia J, Jain D, Ferrell LD. Clinicopathologic features and survival in fibrolamellar carcinoma: comparison with conventional hepatocellular carcinoma with and without cirrhosis. Modern pathology: an official journal of the United States and Canadian Academy of Pathology, Inc 2005; 18(11): 1417-23.

22. Torbenson M. Review of the clinicopathologic features of fibrolamellar carcinoma. Advances in anatomic pathology 2007; 14(3): 217-23.

23. Weeda VB, Murawski M, McCabe AJ, Maibach R, Brugieres L, Roebuck D, et al. Fibrolamellar variant of hepatocellular carcinoma does not have a better survival than conventional hepatocellular carcinoma--results and treatment recommendations from the Childhood Liver Tumour Strategy Group (SIOPEL) experience. Eur J Cancer 2013; 49(12): 2698-704.

24. Mavros MN, Mayo SC, Hyder O, Pawlik TM. A systematic review: treatment and prognosis of patients with fibrolamellar hepatocellular carcinoma. Journal of the American College of Surgeons 2012; 215(6): 820-30.

25. Maniaci V, Davidson BR, Rolles K, Dhillon AP, Hackshaw A, Begent RH, et al. Fibrolamellar hepatocellular carcinoma: prolonged survival with multimodality therapy. European journal of surgical oncology : the journal of the European Society of Surgical Oncology and the British Association of Surgical Oncology 2009; 35(6): 617-21.

26. Ichikawa T, Federle MP, Grazioli L, Marsh W. Fibrolamellar hepatocellular carcinoma: pre- and posttherapy evaluation with CT and MR imaging. Radiology 2000; 217(1): 145-51.

27. Gras P, Truant S, Boige V, Ladrat L, Rougier P, Pruvot FR, et al. Prolonged Complete Response after GEMOX Chemotherapy in a Patient with Advanced Fibrolamellar Hepatocellular Carcinoma. Case reports in oncology 2012; 5(1): 169-72.

28. Patt YZ, Hassan MM, Lozano RD, Brown TD, Vauthey JN, Curley SA, et al. Phase II trial of systemic continuous fluorouracil and subcutaneous recombinant interferon Alfa- $2 b$ for treatment of hepatocellular carcinoma. Journal of clinical oncology: official journal of the American Society of Clinical Oncology. 2003; 21(3): 421-7.

29. Malouf GG, Brugieres L, Le Deley MC, Faivre S, Fabre M, Paradis V, et al. Pure and mixed fibrolamellar hepatocellular carcinomas differ in natural history and prognosis after complete surgical resection. Cancer 2012; 118(20): 4981-90. 